\title{
Early diagnosis of a developing biosystem using acousto-optic imaging
}

\author{
A B Burlakov', A S Machikhin ${ }^{2}$ D D Khokhlov', V I Kuzmin ${ }^{3}$, A F Gadzaov ${ }^{3}$, D \\ L Tytik ${ }^{4}$, S A Busev ${ }^{4}$, V E Kasatkin ${ }^{4}$ and L A Sleptsova ${ }^{1}$
}

${ }^{1}$ Lomonosov Moscow State University, Leninskie Gory, 1, Moscow, Russia, 119991

${ }^{2}$ Scientific and Technological Center of Unique Instrumentation RAS, Butlerova street, 15, Moscow, Russia, 117342

${ }^{3}$ Russian Technological University (MIREA), Vernadsky Avenue, 78, Moscow, Russia, 119454

${ }^{4}$ Frumkin Institute of Physical Chemistry and Electrochemistry RAS, Leninsky Avenue, 31, Moscow, Russia, 119071

e-mail: aalexanderr@mail.ru, demid06101993@gmail.com

\begin{abstract}
Studying the embryo development at an early stage is considered. For this, hyperspectral imaging using an acousto-optical microscope-based system is proposed. Acousto-optic visualization of the early developmental stages of the loach Misgurnus fossilis embryo showed a regular change in the spectral characteristics of different image areas corresponding to the tissues and body fluids, depending on the functional state of the embryo. We show that there is a discrete set of maxima in the optical absorption spectrum with a distance of $20 \mathrm{~nm}$ and $30 \mathrm{~nm}$ between them. Value $20 \mathrm{~nm}$ corresponds to the normal development of biological tissue. The appearance of the value $30 \mathrm{~nm}$ between the maxima of neighboring optical absorption bands indicates the development of pathological processes in the biosystem. Such deviations from the norm are characteristic of germinal tissues, and they are absent in the perivitelline fluid. Thus, we demonstrated that for the early diagnosis of the physiological state of a developing biosystem, special attention should be paid to optical absorption spectra.
\end{abstract}

\section{Introduction}

At present, numerous studies are devoted to the fundamental mechanisms of developmental biology, since changes occurring during lifetime from the moment of fertilization of an ovum to an adult multicellular organism cause many questions related to the formation of a complex multi-level system that is constantly becoming more complex [1-3].

The use of the embryonic model for the research is very promising, since it provides a possibility to analyze the functional features of the various embryogenesis stages, including the initial ones, when within a short period of time many processes that determine the subsequent vital activity of organisms (active cell division, differentiation, morphogenesis) occur. Even the smallest impact in the early development stages may entail the appearance of various delayed effects. In addition, embryonic models have unique advantages for studying the preservation of the completeness of genetic information, which can only be found in early ontogenesis, since many of the most important genes for 
population conservation are expressed during this period, and a change in the regulatory mechanisms of gene expression occurs in the initial period of embryogenesis [4,5].

To study these processes, non-invasive methods that allow not to interfere the vital activity of a living biological system are needed. Spectral imaging is one of such methods [6,7]. It allows quantitative characterizing the spatial distribution of spectral properties. In this research, we demonstrate the effectiveness of this technique for early diagnosis of the developing biosystem. For this purpose, we have assembled an experimental setup based on acousto-optic (AO) imaging add-on module installed on a microscope. We measured and analysed the optical absorption spectra of different areas corresponding to fluids and tissues of the loach embryo and showed that this method may be effectively used to define the state of a developing biosystem.

\section{Experimental setup}

Different approaches and instrumentation are used to obtain spectral images, but the method based on tunable optical filters is most versatile. In comparison to other filters, AO tunable filters (AOTFs) provide a unique combination of features: arbitrary spectral access, high throughput, and the ability of modulating the transmission function, rather high spectral and spatial resolution, small tuning time, while being programmable and totally PC-controlled devices with no need of mechanical adjustment. AOTF is based on anisotropic Bragg diffraction of wideband optical radiation on the ultrasound wave [8]. The central wavelength $\lambda$ of the filter transmission band is defined by the period of ultrasound wave which is driven by a radio-frequency signal and can be controlled electronically.

Fig. 1 shows an imaging system used for the research. The illumination from the light source is collected by the Koehler illumination system. After passing through the specimen, the light is collected by the objective lens, which forms the image of the specimen in the infinity. After passing the tube lens, the light is divided into two paths by means of a beam-splitting prism. One path leads to the binocular tubes, in which the eyepieces 2 are located. Their front focal planes coincide with the back focal plane of the tube lens. Consequently, the eyepieces 2 form the infinity-located image of the specimen for convenient visual inspection. The other path is intended for digital image acquisition. The tube lens translates the image directly onto the recording plane of color camera 3.

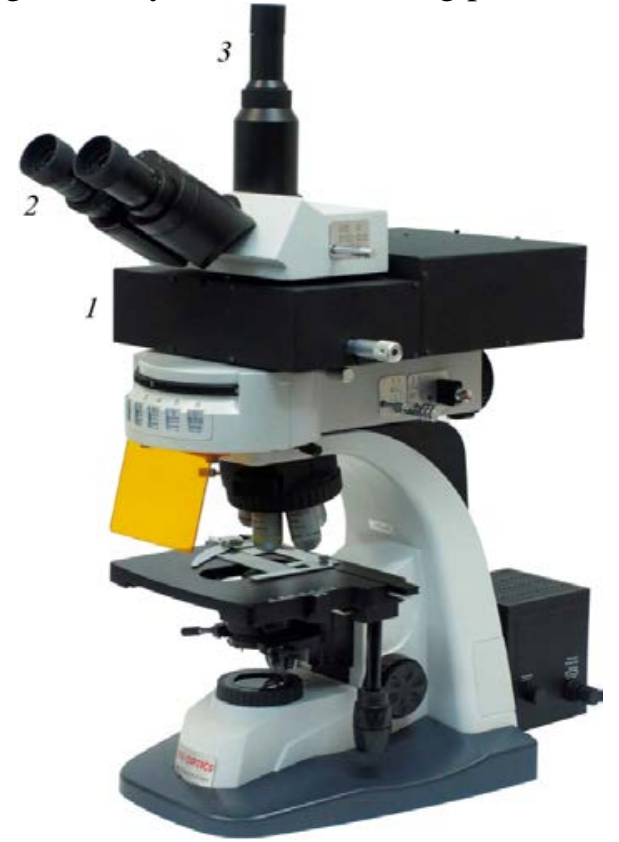

Figure 1. Microscope-based AO spectral imager. 1 - AO spectral imaging unit, 2 - eyepieces for visual observation, 3 - color camera for wideband image registration.

The AOTF-based add-on module 1 is inserted between the microscope body and the trinocular head. It consists of a beam-splitter, an optical coupling system, the AOTF, an imaging objective and a monochrome camera [9]. The device is designed to allow simultaneous visual observation of the 
specimen, color image registration and acquisition of spectral image series necessary for the measurement of the absorption spectra distribution.

Strong chromatic drift and spatial aberrations of the filtered image caused by diffraction light by ultrasound can reach several percent of the field of view and make it impossible to obtain accurate spectral measurements using AOTF images. To overcome this problem we to use the tandem AOTF [10].

For precise measurement of the spectral dependence $I(\lambda)$ in any image point $x, y$, it is necessary to provide repeatable values of the wavelength $\lambda$, intensity $I$ and coordinates $x, y$. That is why, generally spectral imager should have a spectral, amplitude and spatial calibrations, correspondingly [11]. In our case, the specimen may slightly move during a longtime experiments, which leads to the necessity of additional motion correction (Fig. 2).

Spectral calibration is provided using a certified diffraction spectrometer by accurate measurement of the dependence of the selected wavelength $\lambda$ on the frequency $f$ applied to AOTF piezotransducer. To compensate optics vignetting and illumination non-uniformity, a series of background spectral images $B(x, y ; \lambda)$ is acquired. After background correction, correlation techniques are applied to the areas of interest in order to find its offsets $\Delta x(\lambda)$ and $\Delta y(\lambda)$ during the experiment. Only after all these three types of correction are done, the corrected images $I(x, y ; \lambda)$ are used for the spectral measurements.

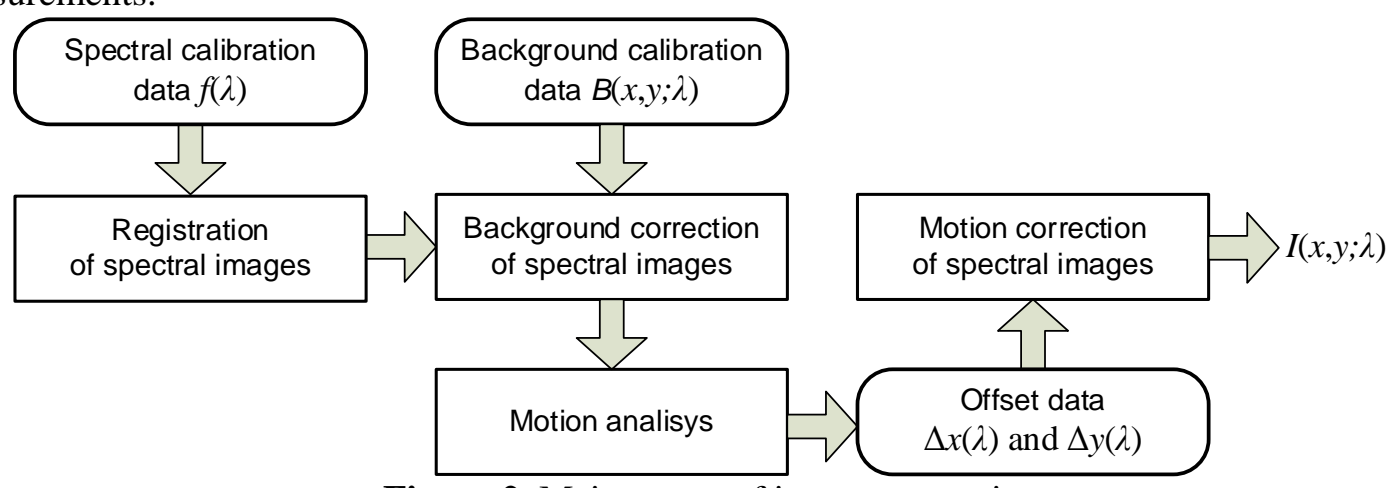

Figure 2. Main stages of image processing.

The experiments were carried with the embryo of the loach Misgurnus fossilis which is considered to be a classical object of developmental biology. Females caught from nature inhabitant were kept in a refrigerator at $4-5^{\circ} \mathrm{C}$. Accelerated maturation of females was performed by hormonal stimulation of chorionic gonadotropin at room temperature, the artificial insemination was performed according to the standard method [12]. The fertilized eggs were thoroughly washed with two portions of fresh water. After that some of the embryos (50 pcs) were placed in an isolated storage with a stabilized temperature of $17^{\circ} \mathrm{C}$. Development stages were determined according to the tables of normal development of the loach $[13,14]$. The embryo of a certain developmental stage was thoroughly washed with two portions of fresh water and placed in the object plane of a microscopic spectral imager. Spectral image series of the developing embryo were registered twice a minute for approximately an hour.

\section{Results and discussion}

Fig. 3 shows one of the spectral images of the loach embryo at 33rd development stage obtained after the correction procedure. The areas used for spectral analysis are highlighted in red. These regions represent different parts of embryo (myotomes, different parts of yolk and perivitelline space).

Fig. 4 summarizes the spectral distributions obtained in different regions of loach embryo. The first feature in the optical absorption spectra for different regions (maxima or their absence) is in the wavelength region nearby $477 \mathrm{~nm}$. Light at this wavelength plays an important role in many physiological processes occurring in all living organisms [15]. For head section (eye area) and yolk in the area of the head section, there is a sharp drop in optical density up to $485 \mathrm{~nm}$. In this case, in different areas of the perivitelline space, a significant increase in the optical density is observed with a 
maximum at $477 \mathrm{~nm}$, followed by a sharp drop in optical absorption to the minimum, at $485 \mathrm{~nm}$. We note that in the region of differentiating myotomes, the optical density in this region of wavelengths does not change. A characteristic feature for all spectra is the greatest change in optical density in the wavelength range from 450 to $590 \mathrm{~nm}$. In the wavelength range of 590-720 nm, insignificant changes in the optical density are observed for all selected regions of the loach embryo.

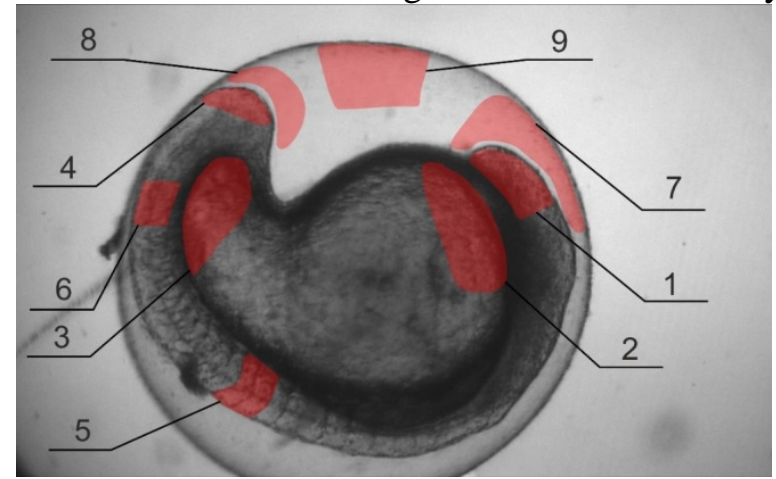

Figure 3. Spectral image of the loach embryo at 33rd stage of development $(\lambda=635 \mathrm{~nm})$. 1 - head section (eye area); 2 - yolk near the head; 3 - yolk near the tail; 4 - tail section; 5 - differentiated myotomes; 6 - differentiating myotomes; 7 - perivitelline space near the head; 8 - perivitelline space near the tail; 9 - perivitelline space.

More detailed analysis of the spectral features for different regions of the loach embryo emphasizes the simultaneous growth of optical density at a wavelength of $500 \mathrm{~nm}$ for all regions of the embryo. In the wavelength region of 580-620 nm, the spectra can be divided into two groups: with a slight change in optical density (perivitelline space and tail region in the tail part of the embryo) and spectra with well-defined maxima (other tissues shown in figures 2 and 4). In the spectral range from 640 to 670 $\mathrm{nm}$, it is possible to conclude about peculiar resonance rearrangements in the tissues of the embryo by the spectra appearance. In the wavelength range $670-720 \mathrm{~nm}$, the spectra are naturally divided into two groups according to the criterion for the presence of a local maximum in the spectrum.

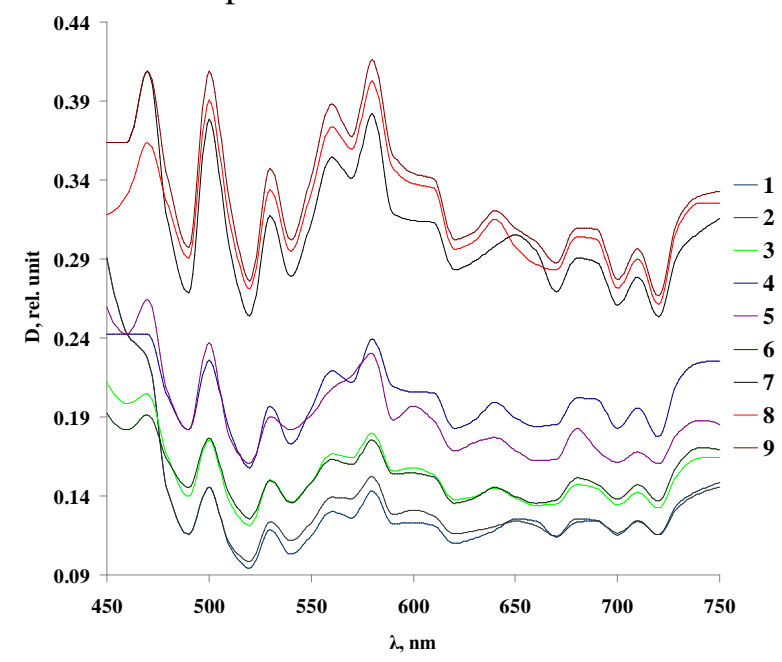

Figure 4. Optical density spectral distributions for different regions. Curve numbers correspond to the region numbers in Fig. 3.

For each spectrum, a pattern was found in the location of the maximum values of the optical absorption intensity in different regions of the embryo, determined by a discrete set of uniform intervals (the distance between adjacent maxima), among which the values of $20 \mathrm{~nm}$ and $30 \mathrm{~nm}$ dominate. The intervals of $20 \mathrm{~nm}$ correspond to regular sequences of uniform strokes known for the scale of characteristic dominant sizes in natural environments $[13,14]$, while the presence of an interval of $30 \mathrm{~nm}$ is associated with the pathological development of a biosystem. Thus, the spectral 
measurements have a clear biophysical interpretation. Further applying advanced techniques for spectral image processing may allow to extract much more morphological data [16,17].

\section{Conclusion}

Our analysis shows that spectral imaging of a developing biosystem is an informative and promising technique. It allows contrast visualisation as well as absorption spectrum measurements in each image pixel. The biggest fluctuations in optical density are observed in the areas of perivitelline space (glycoprotein solution). For the image areas corresponding to the loach embryo tissues (tail section, differentiating myotomes and yolk in the area of the tail section), a stable change in optical density is observed. It is known that the yolk in the tail section is actively consumed for the embryo energy needs and the yolk in the region of the head section is preserved until the late development stages. The difference in spectral characteristics for these areas of the yolk qualitatively confirms its utilization. Thus, the use of an AO spectral imager provides a fast and precise estimation of living system properties as well as a unique opportunity to analyse 61the dynamics of the biochemical processes in a developing embryo by means of processing the time changes of absorption spectra.

\section{References}

[1] Gilbert S 2000 Developmental Biology (Sunderland (MA): Sinauer Associates Inc.)

[2] Cooper G M and Hausman R E 2007 The Cell: A Molecular Approach (Sunderland (MA): Sinauer Associates Inc.)

[3] Heams T, Huneman P, Lecointre L and Silberstein M 2015 Handbook of Evolutionary Thinking in the Sciences (Berlin: Springer) 265-283

[4] Zhiyuan G and Korzsh V 2006 Fish Development and Genetics: The Zebrafish and Medaka Models (Singapore: World Scientific Pub Co Inc.)

[5] Kimmel C B, Ballard W W, Kimmel S R, Ullmann B and Schilling T F 1995 Stages of embryonic development of the zebrafish Developmental dynamics 203 253-310

[6] Li Q, He X, Wang Y, Liu H, Xu D and Guo F 2013 Review of spectral imaging technology in biomedical engineering: achievements and challenges J. Biomed. Opt. 18(10) 100901

[7] Zimmermann T, Rietdorf J and Pepperkok R 2003 Spectral imaging and its applications in live cell microscopy FEBS Letters 546(1) 87-92

[8] Goutzoulis A P and Rape D R 2004 Design and Fabrication of Acousto-Optic Devices (Boca Raton: CRC Press)

[9] Polschikova O, Machikhin A, Batshev V, Ramazanova A, Belov A and Pozhar V 2017 AOTF based optical system of a microscope module for multispectral imaging techniques Proc. SPIE $10592105920 \mathrm{H}$

[10] Machikhin A, Batshev V and Pozhar V 2017 Aberration analysis of AOTF-based spectral imaging systems J. Opt. Soc. Am. A 34(7) 1109-1113

[11] Podlipnov V and Skidanov R 2017 Calibration of an imaging hyperspectrometer Computer Optics 41(6) 869-874 DOI: 10.18287/2412-6179-2017-41-6-869-874

[12] Kostomarova A A 1975 Objects of developmental biology (Moscow: Nauka) 309-323

[13] Jirmunsky A V and Kuzmin V I 1990 Critical levels in the development of natural systems (Leningrad: Nauka)

[14] Fujimoto T, Kataoka T, Sakao S, Saito T, Yamaha E and Arai K 2006 Developmental stages and germ cell lineage of the loach (Misgurnus anguillicaudatus) Zoological Science 23(11) 977989

[15] Villamizar N, Vera L M, Foulkes N S and Sánchez-Vázquez F J 2014 Effect of lighting conditions on zebrafish growth and development Zebrafish 11(2) 173-181

[16] Myasnikov E 2017 Hyperspectral image segmentation using dimensionality reduction and classical segmentation approaches Computer Optics 41(4) 564-572 DOI: 10.18287/2412-61792017-41-4-564-572

[17] Brianskiy S and Vizilter Y 2018 Morphological conditional estimates of image complexity and information content Computer Optics 42(3) 501-509 DOI: 10.18287/2412-6179-2018-42-3-501509 